\title{
Simulation environment design for the investigation of damping and dewing mechanisms in the $12 \mathrm{kV}$ high-voltage switchgear
}

\author{
Qize Pan ${ }^{1}$, Fang Yang ${ }^{1}$, Xiaoliang Tang ${ }^{1}$, Zhi Yang ${ }^{1}$, Shulin Liu ${ }^{2}$ \\ ${ }^{1}$ Qingyuan Power Supply Bureau of Guangdong Power Grid Co., Ltd., Qingyuan, Guangdong 511518, China \\ ${ }^{2}$ Guangdong Lianhang Intelligent Technology Co., Ltd., Guangzhou, Guangdong 510663, China
}

\begin{abstract}
The phenomenon of dampness and dewing inside the switchgear can improve the occurrence of serious equipment faults. Based on it, this paper, starting from the occurrence mechanism of dampness and dewing of the switchgear, summarizes the main techniques that can prevent and control them, and the merits and demerits of them are analysed. After that, based on the mechanism investigation of damping and dewing of $12 \mathrm{kV}$ switchgear, a simulation environment is designed in order to investigate the mechanism of damping and dewing the $12 \mathrm{kV}$ switchgear. Firstly, the entire simulation environment factors are given. Then, the prototype of this switchgear is designed, including base configuration and sensor installation configuration. In addition, the simulation environment of the distribution room, the switchgear with load operating conditions, several commonly used anti-dampness and anti-dewing methods of $12 \mathrm{kV}$ switchgear, and the rotary dehumidifier are designed. Lastly, prospects are given. This paper can provide some references for studying of damp-proofing and anti-dewing of $12 \mathrm{kV}$ switchgears.
\end{abstract}

\section{Introduction}

As a kind of electric equipment, switchgear or switch cabinet has been widely applied to urban power supply and distribution system. The normal operation state of switchgear may be changed due to variation of its internal environment temperature and humidity, as well as air-tightness. When the switchgear is in maintenance, it will be inevitable that damp air from outside will enter into the switchgear, resulting in dampness and condensation phenomenon occurred inside the switchgear. This can easily lead to serious accidents, thus it will affect the power supply reliability level of the whole system will also be reduced ${ }^{[1-3]}$. Currently, there have less research on the mechanism of damping and dewing occurred in the switchgear, among which a few research institutes have demonstrated relevant results. Furthermore, owing to the difference between abroad and China in equipment and operation management, few studies can be searched about the specialized modelling of dampness and condensation and new prevent and control technique for the switchgear.

Chen et al. have conducted numerical simulation research on the dewing phenomenon occurred in several kinds of closed-type switchgears ${ }^{[4]}$. Besides, an antidewing management system for the distribution network switchgear has been developed in ${ }^{[5]}$, based on the environment temperature data and humidity monitoring data in the location of the distribution network switchgears. On the whole, the physical models established in most of current researches on antidampness and anti-dewing inside the switchgear are excessively ideal, thus they are very different from the actual ones. This is mainly because the internal structure of the switchgear is complicated with lots of components.

\section{Mechanism of dewing in the cabinet}

\subsection{Dewing cause}

During the dewing process inside the switchgear, because the water vapor in the cabinet has exceeded its maximum containment capacity, it will be liquefied into water on the surface of the internal cryogenic object immediately after its temperature reaching the dew point temperature, which is called dewing phenomenon.

Generally speaking, the occurrence of dewing inside the switchgear depends on two conditions: first, the internal ambient humidity of the cabinet, $F(\% \mathrm{RH})$, and the second one is the change of the ambient temperature inside cabinet, $t\left({ }^{\circ} \mathrm{C}\right)$. The higher the ambient humidity in cabinet, the more easily the dewing occurs; and the bigger the variation of the ambient temperature in the cabinet, the more easily the dewing occurs. Therefore, under certain temperature conditions, when the relative humidity in the air is higher, the temperature of dew point will be closer to the ambient air humidity, and the dewing phenomenon will happen more easily; when dewing occurs in the cabinet, the dew point temperature is always lower than the ambient temperature, regardless of the temperature in the air.

\footnotetext{
* Corresponding author: Qize Pan, powertoyou@163.com
} 


\subsection{Major dehumification measures}

The first one is the dry dehumidifier, in which the internal temperature of the switchgear is improved via heating, so as to improve the ability of air to dissolve water and reduce relative humidity, thus the condensation is not easy to happen ${ }^{[6,7]}$.

The second one is the condensing dehumidifier, which can overcome the demerits of heating dehumidifier, the condensing dehumidifier has been proposed, in which the chiller is took as the core component.

The third one is the dry-air dehumidifier, in which the rotary dehumidifier is selected as the dehumidification apparatus. Through the dehumidifier, the dry air is continuously transported to the switchgear to drive away the humid air in the cabinet.

Based on the descriptions above, the comparison of the three major dehumidifiers is as follows.

a)Heating dehumidifier: its equipment is heater. The wet gas in the cabinet is not completely discharged, but only the ability to accommodate is improved after the air temperature is raised, and the relative humidity is reduced as well; there is a dead zone of anti-dewing and anti-dampness because of the installation position and the limit of power size and air circulation.

b)Condensing dehumidifier: semi-conductor dehumidifier is as its equipment. The air in the cabinet is condensed into water and then discharged. Obvious demerits: a low efficiency in low temperature and high humidity; risk of water leakage; damage IP protection level; and low resistance to small animal; the minimum diameter of drain pipe is not less than $6 \mathrm{~mm}$, which is generally $8-10 \mathrm{~mm}$, and is much larger than that of highvoltage switchgear IP3X protection grade $2.5 \mathrm{~mm}$.

c)Dry air dehumidification approach: rotary dehumidifier is as its equipment. The dry air is transported to the cabinet to drive out the damp air in the cabinet; seen as a process of air exchange, with a fast speed of dehumidification and high efficiency. High dehumidification ability; not only can be used for antidampness and anti-dewing of the switchgear, but also can improve the humidity environment of the electric room; the control mode is transformed from decentralized control into centralized control, thus no power cut is needed in maintenance of the cabinet without dehumidification element, and the safety is high.

\section{Simulation environment design}

\subsection{Simulation environment factors}

The simulation environment factors for investigation of damping and dewing mechanisms of the $12 \mathrm{kV}$ high voltage switchgear including: switchgear physical model simulation, power distribution room simulation, operation condition simulation, and dehumification device operation simulation, as illustrated in Fig. 1.

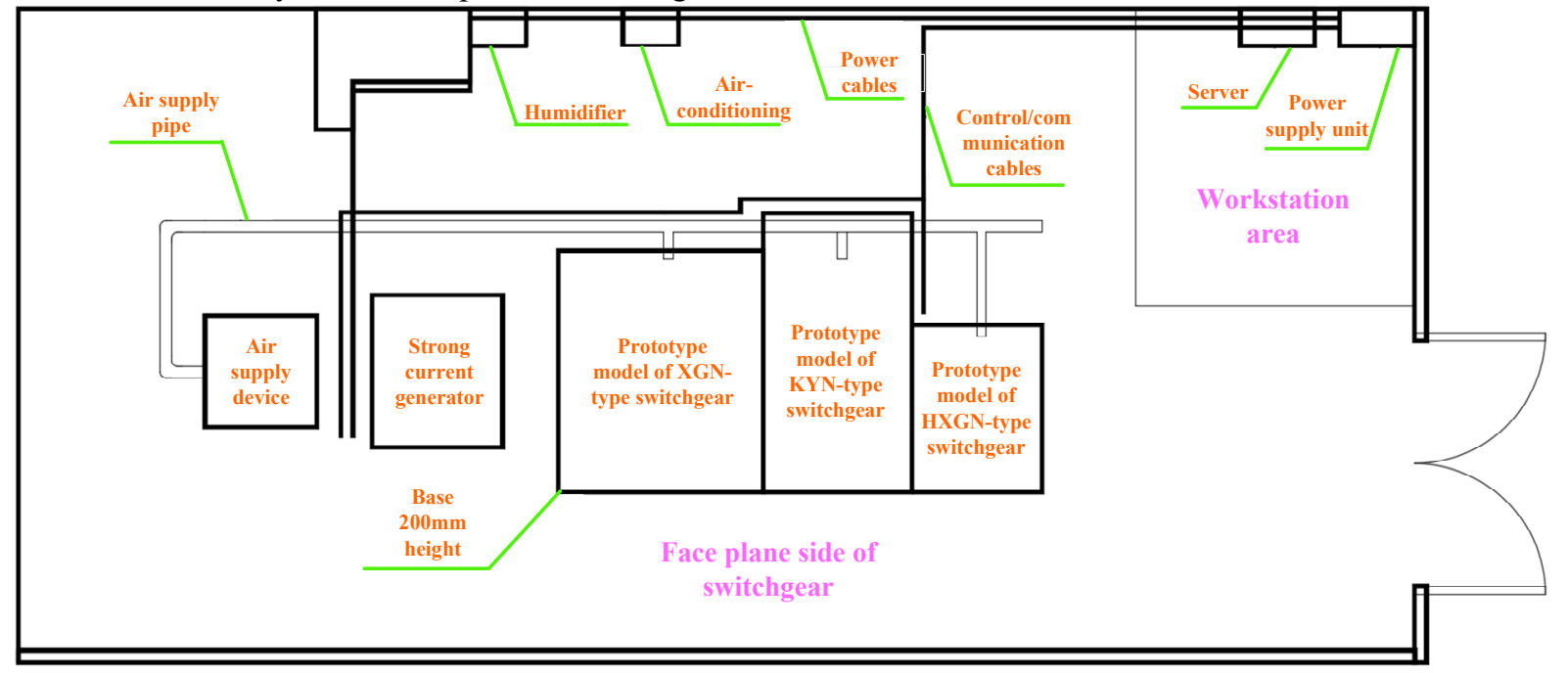

Fig. 1. Entire framework design of the simulation environment.

\subsection{Prototype model design of switchgear}

The physical model of the switchgear shall be designed and manufactured in accordance with the technical specifications of the distribution grid switchgear of China Southern Power Grid Corporation, including component configuration, external dimensions, and IP class, and shall be specially designed according to the requirements of the base configuration and sensor installation configuration. The specific configurations are shown as follows. For the XGN-type, KYN-type and HXGN-type switchgears, their dimensions (width * depth * height / $\mathrm{mm}$ ) are $1100 * 1300 * 2650$,
$800 * 1500 * 2360$, and $700 * 900 * 2000$, respectively; rated voltage are all $12 \mathrm{kV}$; rated current are $1250 \mathrm{~A}, 1250 \mathrm{~A}$, and $630 \mathrm{~A}$, respectively; cabinet sealing requirements are as follows: degree of enclosure protection are IP3X, IP4X, and IP3X, respectively, and the permissible gap width are $2.5 \mathrm{~mm}, 1.0 \mathrm{~mm}$, and $2.5 \mathrm{~mm}$, respectively.

The external dimensions, rated voltage, rated current, and cabinet seal are designed according to the power grid specifications. The purpose is to allow the study to conform to the actual structure of the grid switchgear.

According to Fig. 1, the base configuration requirement is to simulate the condition of the cable trench. The switch cabinet is mounted on the base and simulates its installation on the cable trench. The base is 
$200 \mathrm{~mm}$ high and the middle is empty. The inside can be filled with sponges that are saturated with water. The simulation of wet cable ducts continues to influence the humidity of the switch cabinet. These humid gases enter the switchgear through the gaps of the switchgear, and the actual switches. The installation environment of the cabinet is the same.

The requirements of sensor installation and configuration are for real-time and continuous collection of the three compartments of the switchgear, affecting the three elements of condensation: the distribution and changes of temperature, humidity, and air pressure. It is a large number of data sources that need to be used in the study. Infrared thermography thermometer, a $160 * 120$ pixel thermal imager that can provide thousands of temperature measurement points, can compensate for the lack of sensors, or make up for the lack of installation location limitations.

\subsection{Simulaiton environment design of the power distribution room}

The simulation of power distribution room environment includes power distribution room, humidifying device and air conditioning device; The power distribution room can be equipped with equipment such as the physical model of the lower three sides of the switchgear. The width is $4000 \mathrm{~mm}$, the length is $9000 \mathrm{~mm}$, and the height is $3800 \mathrm{~mm}$. Other requirements are the same as the general power distribution room requirements.

The role of humidification device is to provide adjustable humidity environment, use of intelligent ultrasonic humidification equipment, the maximum hourly humidification capacity of $3 \mathrm{KG}$, the amount of humidification can be adjusted according to need to achieve the regulation of power distribution room humidity; The function of the air conditioning device is to provide an adjustable humidity environment, and the inverter-driven air conditioner is selected to minimize the humidity fluctuation.

The simulated design of the power distribution room environment can achieve extreme environments of high temperature, high humidity, low temperature and high humidity, or other close-to-real environments that need to be studied.

\subsection{Simulaiton environment design of operating conditions of switchgear with loads}

The simulation of the switchgear on-load operating conditions is realized by a large current generator. The input power of the large current generator is AC400, the output current is three-phase, and the maximum is 2000A. The output current can be adjusted to the rated current of the switchgear or any current. The simulation of the operating conditions of the heating effect of the switchgear with load is realized.

The large current generator is connected to the copper bar led by the busbar room of the switchgear cabinet through cables. The connection mode is threephase connection. Each switchgear is short-circuited in the cable room, so when the switch is closed, it can be realized. The simulated simulation of the on-load operation with the actual operation reflects the effect of heat generation on the formation of condensation when the switchgear is in operation.

\subsection{Simulaiton environment design of several anti-damping and anti-dewing measures commonly used in the $12 \mathrm{kV}$ high voltage switchgear}

Several common anti-condensation and moisture-proof methods for switch cabinets are heating method and condensate drainage method. The principle of the heating method to prevent condensation and moisture is to heat the electric heating element to increase the temperature of the air in the cabinet, thus improving the ability of the air to contain water molecules and reducing the partial pressure of the water molecules in the air, thereby reducing the relative humidity of the air. Anticondensation and moisture-proof are realized; this method does not reduce the moisture in the air inside the cabinet, so when the temperature drops, condensation is still easy to occur.

By installing heating plates in the compartments of the switch cabinet and configuring the automatic controller, it is possible to simulate the effectiveness of condensation control of the switchgear only by heating plates;

For the Condensate Drain Method, its principle of anti-condensation and moisture proof is through the Peltier effect of semiconductor devices. In a semiconductor with direct current, the cold junction temperature can fall below the air condensation point, and the moisture in the air can be condensed into liquid water., And then the water out of the cabinet, thus reducing the air's water molecule content, reducing the water molecules in the air in the sub-pressure, while reducing the relative humidity of the air, but also reduce the absolute humidity, to achieve anti-condensation dew moisture; This method requires liquid water to be produced in the cabinet and then discharged. When the drain tube is clogged, there is a danger of water accumulation in the cabinet. At the same time, the presence of the drain tube destroys the protection level of the switch because the diameter of the drain tube is usually $6-10 \mathrm{~mm}$., is much larger than the $1-2.5 \mathrm{~mm}$ protection requirements of the switch.

By installing the condensation dehumidification device in each compartment of the switchgear cabinet and automatically starting and stopping according to the setting value, the effect of condensation prevention and control by using the condensation drainage method can be simulated.

A new type of anti-condensation and moisture-proof method is simulated. Through research and analysis, the dry gas replacement method is an innovative method for preventing and controlling moisture condensation in switchgear cabinets. The basic principle is to use dry gas sources to make dry gas, and then to pass through the air-supply tubes after boosting the temperature. Into the 
switchgear, the cabinet will be out of the humid air, the formation of replacement effect, with centralized gas supply, high efficiency, speed, dehumidifier device in the switch cabinet, maintenance without power and other advantages.

Through the installation of dry gas source and air supply pipe in the power distribution room, the branch air inlet pipe is installed at the bottom of each switch. The operation of the air source device automatically starts, boosts pressure, adjusts the temperature and stops according to the setting value, and the analog switchgear cabinet can be dry. Gas replacement method for condensation control effect.

\subsection{Rrotary dehumidifier design}

The rotary dehumidifier is selected as the equipment of dry gas source, thus the basic structure of the dry gas source equipment is shown in Fig. 2. Here, the air to be treated is sent to the circulating air inlet, and then the air becomes dry air after the water in it is absorbed by the wheel. Then, the dry air is sent to the air pressure regulating and temperature adjusting device. In this device, the air pressure is raised to facilitate transportation, and at the same time, the temperature is adjusted to meet the requirements of anti-dewing and anti-damping of the switchgear, and the operation temperature. The dry gas from the device is controlled by the switchgear's air supply valve (VS1) to supply gas to the switchgear. These gases will be dispersed from the switchgear vent or gap to the indoor area, and then enter through the circular inlet to form a cycle, so that the moisture in the switchgear and indoor air is absorbed and dried. The indoor air supply valve (SV2) will be used to supply air in order to regulate the indoor environment without the need for sufficient gas or gas source in the switchgear. The water after the wheel absorption is discharged outside through the regenerated exhaust air.

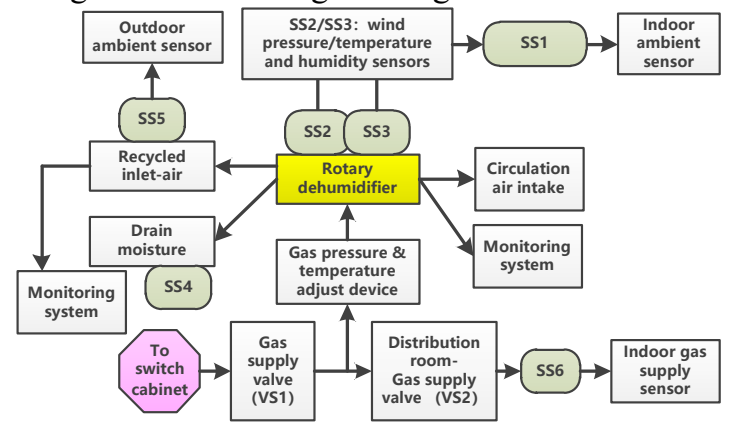

Fig. 2. Structure of dry air source equipment and design of dry air entering into the cabinet via nozzles.

At the present stage, there are few studies on the characteristics of air flow in the cabinet, which leads to dead zone of anti-damping and anti-dewing when adopting the traditional dehumidification method. The advantages of dry air dehumidification lie in the following aspects: it can make full use of the air pressure of the gas-supply system, in order to make the air in the cabinet forced to flow, such that achieving the purpose of dry air circulation in the whole cabinet and the goal of no dead zones; by arranging the nozzle angle, we can drive the air to generate rotary flow so as to enhance the efficiency of dehumidification, so that it can supply gas to a specific narrow part and realize the damp proofing and anti-dewing of the whole cabinet. To achieve this key goal, we can compare and analyse the monitoring data collected by the sensors in the cabinet, so as to find out the best nozzle and the best installation angle.

It still needs to be focused on the development of a monitoring and control system for the damp proofing and anti-dewing of the switch cabinet. Here, this developed system is employed to monitor the operation of gas source and gas distribution system. Besides, according to the collective data of temperature and humidity inside the cabinet, the system can be used to calculate the dew point and give the control value, in order to control the temperature and humidity in the cabinet, and make them away from the dew point. At the same time, this system should also possess the functions of remote metering, remote signalling, remote regulating, and remote controlling, in order to realize the application of micro signal on mobile devices, and to inquire the data to receive early warning information at any time. Specifically, the monitoring and control system is basically composed of server, software, controller, and communication network, thus it can finally realize the intelligent management of the whole device.

In order to simulate the actual operation environment, the experimental environment should be built as follows:

a) the distribution room: with area $\geq 26 \mathrm{~m}^{2}$, storm height $\geq 3.2 \mathrm{~m}$, length* width $\geq 4 \mathrm{~m}^{*} 6 \mathrm{~m}$, and equipped with three-phase power supply with capacity of not less than $30 \mathrm{~kW}$;

b) ambient conditioning equipment: equipped with 1 humidifiers, $\geq 30 \mathrm{kG}$ per day, equipped with refrigeration/heating dual purpose air conditioner, and equipped with infrared heater, $\geq 5 \mathrm{~kW}$ and the output power is adjustable;

c) physical model of switch cabinet: can realize the installation and adjustment of the thermal infrared imager, combined temperature and humidity sensors, smart controller, heating plate, dehumidifier, communication cable, dry gas control valve;

d) simulation of the operation current: equipped with a large current generator, with the output current less than 1500A;

e) the data acquisition system: the temperature \& humidity sensors should be disturbed in each isolated room of the switchgear, and the infrared thermography should be allocated.

In addition, the data acquisition and analysis software should be allocated. Concretely, the sensors in the switchgear collect the data of temperature and humidity, which are used for continuous calculation and analysis, realizing centralized control and adjustment. Moreover, this monitoring software should adopt $\mathrm{B} / \mathrm{S}$ architecture, in order to achieve data sharing and remote cooperative working, and finally improve the investigation efficiency.

\section{Prospects}

In the future, there will be great space for the research on the prevention and control of damping and dewing in the 
switchgear, including compression-condensation method, atmospheric pressure condensation, chemical adsorption and physical adsorption methods. Among them, the wheel dehumidification method in the physical adsorption method has the advantages of large dehumidification, less maintenance, no environmental influences, and small volume. But in order to obtain the ideal practical gas source, we need further research in the aspects of wet load calculation, turning wheel design and selection, outlet air pressure improvement, temperature control, humidity control, smart control, energy saving and so on. And besides, we need to combine smart grid technology, knowledge automation technology ${ }^{[8]}$, grey system technology ${ }^{[9,10]}$, internet of things technology, smart diagnosis technology [11], AI technology, deep learning ${ }^{[8]}$, big data technology, and cloud computing with other switchgear damp-proofing and anti-dewing techniques in the future, in order to realize the purpose of intelligent prevention and treatment for the highvoltage switchgears in the distribution networks.

\section{Conclusion}

This paper presents the simulation environment design for the investigation of damping and dewing mechanisms of the $12 \mathrm{kV}$ high voltage switchgear. It is a common phenomenon that the switch cabinet is exposed to moisture and condensation. It is of great significance to study the mechanism of the switch cabinet and ensure the safe and stable operation of the power grid. Through the discussion of the simulation environment of the switch cabinet condensation mechanism, this paper designed the overall program, elaborated the design requirements and functional characteristics of each subprogram, which can be used as a practical reference for the study of similar projects.

However, there still has a long way to go for the research on damp proofing and anti-dewing of the switchgear. To address it, we argue that in the future we can take steps to combine the theoretical research and device research with practical application verification. First, we carry out modelling of the mechanism of switchgear's dampness and condensation; then, based on it, we combine with new dehumidification technologies, to carry out the new technology research and device development, to develop new technology and new devices suitable for the switch cabinet's moisture-proof and anti-condensation; finally, we combine with professional manufacturer's strength, in order to carry out application research on data collection and analysis, data mining, and smart control technology, and finally we can provide technical support for accurate modelling, reliable moisture-proof, anti-condensation and intelligent control.

\section{Acknowledgement}

This paper is supported by the Science and Technology Projects of China Southern Power Grid (Grant. GDKJXM20162530(031800KK52160015)).

\section{References}

1. Y.Q. Li, Z.H. Wang, C.M. Mei, et al. Reason analysis of one dewing flashover accident in switchgear \& its preventive measure, Shanxi Electric Power, 2009, 37(4): 51-53.

2. W.Q. Zeng. Study and practice of anti-condensation technology for high-voltage switchgear, Metallurgical Power, 2016, 10: 49-50.

3. J. Lu, L.F. Cheng, T. Yu. Key technologies study of smart low-voltage regulation control system in distribution area, The Journal of New Industrialization, 2016, 6(11): 35-47.

4. R. Chen, X. Yan, W. Yan, et al. Numerical situation study of dewing phenomenon in switchgear, Resources Economization \& Environmental Protection, 2015, 11(5): 21, +23.

5. X.D. Li, Z.X. Chen. A kind of management system of anti-condensation for distribution network switch cabinet, Electrotechnics Electric, 2016, 23(9): 58-61.

6. J. Luo, Z.P. Pi, H.W. Luo, et al. The composition of high voltage switchgear prevent condensation system and practices, Modern Industrial Economy and Informationization, 2015, 5(21):20-22.

7. J.G. Liu. Impact of relative air humidity and dewing on mid-set switchgear and the strategy, Electric Engineering, 2016, 37(2): 26-28.

8. L.F. Cheng, T. Yu, X.S. Zhang, et al. Cyberphysical-social systems based smart energy robotic dispatcher and its knowledge automation: framework, techniques and challenges. Proceedings of the CSEE, 2018, 38(1): 25-40.

9. L.F. Cheng, T. Yu, G.P. Wang, et al. Hot spot temperature and grey target theory-based dynamic modelling for reliability assessment of transformer oil-paper insulation systems: A practical case study. Energies, 2018, 11(1), 249.

10. L.F. Cheng, B. Zhou, D.H. Cai, et al. Lifetime assessment and optimized maintenance system of transformers based on the HST model. Lecture Notes in Electrical Engineering, v 334, p 417-430, 2015.

11. L.F. Cheng. Design and research on key technology of intelligent energy-saving and diagnosis device based on dedicated users. Master Thesis, South China University of Technology, Guangzhou, China, 2015. 